\title{
Significance of Mobile Ad-hoc Networks in the Growth of Cloud Based Services
}

\author{
Aniket Deshpande* \\ Faculty of Engineering and Technology, Mewar University, Chittorgarh, Rajasthan, India. \\ *Tel: +91-99679-99779; Email: anik.deshpande@gmail.com \\ Manuscript submitted November 26, 2014; accepted June 25, 2015. \\ doi: 10.17706/ijcce.2016.5.3.222-228
}

\begin{abstract}
Mobile Ad-hoc networks have gained considerable popularity in the past few decades. It is because; these networks facilitate secured, integrated, and cost effective wireless combination. Recently, studies have established an approach that mobile ad hoc networks may commendably support the growth of cloud-based services, as the operations of both of these networks are comparable. This manuscript will assist in identifying the role and performance of mobile ad hoc networks in the growth of cloud based services, while recognizing different issues and challenges. Mobile Ad-hoc performance limitation and challenges may play a major role in affecting the performance and growth of cloud based services. In this regard, different techniques and methods will also be analyzed and encapsulated in the research study so as to assure the reliability of cloud-based services' operations.
\end{abstract}

Key words: Ad-hoc networks, cloud-based services, SaaS, application and network performance visibility, monitoring and optimization for cloud services, wireless networks.

\section{Introduction}

The purpose of this paper is to demonstrate the significance of mobile ad hoc networks in the growth of cloud-based services. It has been revealed from the evaluation of researches and studies, which were carried out by R. Hussain [1] that a MANET (mobile ad hoc network) is an incessantly self-configuring infrastructure-less network of mobile devices, which are linked without wires. On the other hand, S. Al-Omari [2] has claimed that a MANET is collection or set of different independent mobile users that communicate over wireless links. It is a fact that nodes are usually mobile, but the network topology can be changed unpredictably and rapidly over time. It has been documented in the researches of R. Gupta [3] that mobile ad hoc networks are playing a major role in the growth and development of cloud based services. It is due to the fact that the operations of cloud technology are quite similar to the functions of ad hoc; hence mobile ad hoc supports the growth of different cloud based services. In accordance with the perceptions of G. Kirby [4], the operations of cloud depend on the sharing of resources, in order to attain coherence and efficiency, which is related to the functions of mobile ad hoc networks. Therefore, it can be asserted that ad hoc is commendably facilitating the growth of cloud-based services. The proceeding paper incorporates the brief evaluation of the term "cloud based services". Moreover, this manuscript will also assist in inspecting the role of ad hoc networks in the growth of cloud based services, while examining the performance limitation or challenges of ad hoc networks in attaining the growth potential of cloud based services. Different solutions and techniques, to overcome the identified, will also be investigated in this research. This study will recognize the role of mobile ad hoc networks in enhancing the growth of cloud services, in 
order to provide financial benefits to the companies. It is expected that both, ad hoc networks and cloud-based services, may play a commendable role in achieving the concept of smart cities or smart governance in the emerging world. The collective potencies of these networks will also be assessed in this research study.

\section{Introduction to Cloud Based Services and Role of Mobile Ad-hoc Networks in Their Growth}

\subsection{Cloud Based Services}

According to R. Gupta [3], the term "cloud based services" refers to the resources, services, or applications, which are made available to the users on demand. It is important to notice that the services are provided to the clients through Internet from the servers of cloud computing providers. In the current era, organizations and businesses have transformed their business operations from traditional computing to cloud based computing approaches. It is due to the fact that cloud based services or computing practices enable the businesses to increase and enhance their capacity, improve their functionality and operations, in an efficient and cost effective manner. According to G. Mcgilvary [5], cloud based services utilizes a virtualized software model and enables the companies to share their networking, storage, as well as their physical services with their desired destinations. It has been contended by S. Al-Omari [2] that cloud technology possess various service models, which are usually deployed in the organizations, after examining their needs. Some of the most prominent service models of cloud may include SaaS (software as a service), PaaS (platform as a service), IaaS (Infrastructure as a service), and CaaS (communication as a service). All of these model of cloud computing plays a substantial role in saving the cost of enterprises and businesses, improving their flexibility and scalability, reliability of operations, and overall accessibility of work related information.

On the other hand, cloud based services also comprise different issues and vulnerabilities, in terms of continuous evolution and security of the network. It has been declared by G. Kirby [4] that these issues may considerably impact or influence the performance and integrity of organizational operations; hence results in devastating outcomes. Thereby, it is considered as one of the utmost responsibilities of the companies to identify and overcome the issues, in order to assure the reliability of operations. R. Gupta [3] has declared that one of the major challenges, which are associated with cloud based services is continuous evolutions or advancements in the requirements for storage, networking, and interfaces. Dynamic nature of cloud based services has become one of the biggest threats for companies, as they have to continually transform their operations, in accordance with the evolutions. According to G. Mcgilvary [5], apart from potential risks and vulnerabilities, cloud computing or cloud based services can be considered as one of the most effective solutions for businesses, in terms of cost saving, higher efficiencies, enhanced integrity, convenience, and improved accessibility.

\subsection{Role of Mobile Ad-hoc Networks in Growth of Cloud-Based Services}

It has been established from the assessment of researches, which were conducted by S. Taneja [6] that mobile ad hoc network (MANET) is a group or collection of independent network mobile devices, which are linked over several wireless links. According to S. Al-Omari [2], it works on a constrained bandwidth, having dynamic network topologies, which are often changed. In this regard, R. Gupta [3] has presented an approach that the increasing use of wireless portable devices, like laptops and phones has increased the usage of mobile ad hoc networks (MANET), as it supports wireless communication amid multiple devices. It is due to the fact that MANET is a self-configuring network, which does not need any fixed or pre-existent infrastructure. This feature plays a vital role in minimizing or reducing the deployment cost and time. Every single node in this node is independent to move, which enables the network to change its topology. These 
infrastructure-less and virtual mobile nodes dynamically form routes amid themselves; hence results in a wireless network. After examining all aspects and characteristics of this network, R. Gupta [3] has stated that mobile ad hoc networks may play an incredible and inevitable role in the growth of cloud based services. R. Hussain [1] has declared that primary purpose of cloud based services is to reduce or completely eliminate the requirement of network infrastructure. In this regard, MANET may commendably assist the companies in establishing their integrated and sustainable cloud based services. It is due to the fact that MANET does not possess fixed routers, specialized hardware, or central servers. The communication amid network nodes is conducted using the help of wireless connectivity. Thereby, it can be avowed that MANET may play a primary role in the growth of cloud-based services. Another major objective of cloud-based services is to reduce the reliance of companies and organizations on conventional energy resources. In this account, mobile ad hoc may imperatively support the companies, as the mobile hosts are considerably small and light in weight. In addition to this, they do not require conventional power resources, as they can be easily operated with the help of small batteries. According to S. Taneja [6], mobile ad hoc is one of the most reliable and effective approaches, which can be adopted for the growth and development of cloud based services.

\section{Performance Limitation of Ad-Hoc Networks in Achieving Growth Potential of Cloud-Based Services}

\subsection{Inadequate Performance of TCP (Transmission Control Protocol)}

Apart from large number of benefits, ad hoc networks possess various limitation and challenges, in terms of its performance and privacy. These challenges of ad hoc networks may noticeably influence the growth potential of cloud-based services. One of the major performance issues, which are related to ad hoc networks, may include the inadequate performance of TCP (transmission control protocol). Different loopholes and flaws in the performance of TCP play a major role in affecting or degrading the performance of mobile ad hoc networks. It has been established by R. Gupta [3] that, when such errors take place, nodes move randomly, supporting to forward or transport data packets, in order to enable communication amid nodes, instead of forwarding the data packets within the range of wireless transmission. This situation often results in the failure or routing malfunctioning; hence results in the loss of data packets. In accordance with the studies and researches, which were carried out by G. Mcgilvary [5], such incidents affect the integrity and reliability of communication practices and may affect the operations of organizations.

\subsection{Security and Privacy Issues}

Another issue, which is associated with the performance of ad hoc networks, may include security and privacy risks. It has been inspected from the assessment of researches, which were accomplished by G. Kirby [4] that ad hoc networks are more likely to face different types of attacks and security related issues. Primarily, ad hoc networks have to bear routing attacks, which plays a major role in affecting the integrity of routing process. Routing attacks can be classified into four different kinds, which are further divided into two major types, including resource consumption attacks and routing disruption attacks. These attacks are done using different techniques, most prominently, through modification, fabrication, and impersonation. Apart from resource consumption attacks and routing disruption attacks, ad hoc networks also bear some special kinds of attacks, including black hole attacks and wormhole attacks. According to R. Gupta [3], these attacks play a significant role in impacting the process of routing; hence results in disruptive communication due to loss of data packets.

\subsection{Other Limitations and Challenges in Ad-hoc Networks' Performance}

S. Al-Omari [2] has contended that such challenges and limitation may considerably impact the potential 
growth of cloud based services. It is due to the fact that different interruptions and disruptions, like loss of data packets may affect the continuity of cloud's operations. It has been contended by S. Taneja [6] that mobile Ad-hoc networks incorporate the traditional issues of wireless networking and wireless communication. Similar to wireless medium, ad hoc networks also possess the vulnerabilities of reliability and security. More so, ad hoc networks also acquire asymmetric and time-varying propagation properties. Precisely, because of having multi-hop nature and having restricted fixed infrastructure, mobile ad hoc has to face number of performance and security issues. In accordance with the studies of G. Mcgilvary [5], various other issues are associated with the performance vulnerabilities of mobile ad hoc networks. These may include unpredictable network topological changes, irregular power consumption, interference, fading, propagation path loss, as well as variable and dynamic wireless link quality.

\section{Techniques to Overcome the Limitations}

\subsection{End-to-End Approach}

One of the major issues or challenges in ad hoc performance, which have been identified in the preceding section, may include malfunctioning or inadequate performance of TCP. Therefore, one of the major solutions for this problem is to detect, identify, and respond to the inoperative or out-of-use packets delivery events. It is due to the fact that these are the major reasons behind frequent and unstoppable route changes. It has been asserted by S. Tanjea [6] that transforming and amending TCP (transmission control protocol) to enhance its functionality in wireless networks has been a venerable problem for researchers and scientists. In this regard, wide range of methods and techniques has been proposed, in order to improve and enhance the performance of TCP in mobile ad hoc networks. Amid all of identified methods, end-to-end approach has been found to be more effective and efficient, which controls the changes at the receiver-end as well as sender-end. In addition to this, end to end approach also plays a primary role in offering flexibility for backward compatibility. G. Kirby [4] has declared that end-to-end approach plays an indispensable role in maintaining and sustaining the end-to-end TCP semantics. End-to-end approach is nothing more than the end-to-end assessments of the network, in order to identify, detect, and measure channel error, route change, disconnection, and congestion. It has been observed that the detection plays an imperative role in triggering the corresponding control actions; hence results in more adequate and sustainable operations of ad hoc networks.

\subsection{Solutions for Security and Privacy Issues}

In order to assure the privacy and security of the ad hoc networks, various methods and techniques have been proposed by scientists and researchers. Some of the most prominent methods may include trust-level metric, secure neighbor verification, randomize message forwarding, end to end feedbacks, watchdog, activity based overhearing, reputation based solutions, nuglets and data dispersal. In accordance with the views of S. Al-Omari [2], these methods play a major role in detecting, preventing, as well as reducing these security and privacy issues, which often takes place in ad hoc networks. Thereby, it is essential for the companies and organizations to assure the scalability, cohesiveness, as well as the reliability of their ad hoc based cloud services by adopting these security measures, against potential malware, viruses, and attacks. In other words, these security measures may commendably help and assist the companies, which are going to implement and deploy ad hoc based cloud services, in establishing highly secured, protected, and sustainable organizational operations, by eliminating the risk of potential attacks and vulnerabilities.

\section{Leveraging the Ad-hoc Networks to Scale up the Growth of Cloud-Based Services and Its Impact on Company's Revenues}

The foregoing manuscript has critically and profoundly discussed the role of ad hoc networks in the 
growth and development of cloud based services. It is quite evident from the discussion that ad hoc networks possess undeniable significance in improved functions of cloud based services. Recent reports, which have been presented by G. Mcgilvary [5], have revealed the fact that ad hoc networks may play an incredible and inevitable role in scaling up the growth of cloud services. One of the major reasons behind this approach is the similarity amid the operations of ad hoc and cloud. In accordance with the perceptions and views of S. Taneja [6], ad hoc networks increase the mobility of cloud based services, which results in more improved and profitable operations of the companies. Therefore, it is considered as one of foremost responsibilities of the researchers to find new ways and methods, in order to leverage the performance of ad hoc networks, as this feature may scale up the growth of cloud based services. According to R. Hussain [1], various security techniques or solutions as well as performance improvement approaches have been discovered, in order to scale up the growth of cloud based services through ad hoc. It has also been assessed that wide range of protected routing protocols have been development, in order to assure the correctness and reliability of ad hoc routing protocols. These integrated features of ad hoc may result in increasing and strengthening the growth of cloud based services. It has been investigated from the in-depth analysis of approach, which was proposed by S. Al-Omari [2] that such features may play an appreciable role in improving and stabilizing the revenues and profitability of the businesses and companies. It is because of the fact that smooth and hassle free deployment of highly protected and wireless cloud based services will assist the companies to control or reduce their operational cost; hence resulting in competitive advantages.

Cloud based services consumes relatively lower power or electricity, which may imperatively impact the economic conditions of the company. In other words, ad hoc networks may offer great opportunities to the cloud based environment to reduce its power consumption, which is expected to result in lower operational cost of the business [3]. Ad hoc based cloud services also offer an opportunity to the enterprises to improve their information accessibility and availability, which ultimately results in the improved operations of the businesses. These improved business operations eventually results in higher revenues, profitability, repute, as well as long term sustainability of business [1]. Most prominently, scaled up growth of cloud-based services, due to ad hoc networks, may also result in higher or improved mobility. Improved mobility may assist the businesses and organizations to acquire their desired information or data, in an efficient, convenient, cost effective, and adequate manner. End result of all of these aspects may improve the overall revenues and economic conditions of the company. After considering all of these characteristics, it can be averred that leveraging of ad hoc networks commendably supports the growth of cloud based services; hence results in the commendable impacts on the revenues of the organizations.

\section{Role of Ad-hoc Networks and Cloud Based Services in Achieving the Concept of Smart Governance and Smart Cities}

It has been divulged from the investigation of researches and approaches, which were presented by G. Mcgilvary [5] that both ad hoc networks and cloud based services may commendably improve and enhance the overall functionality and integrity of cities, countries, organizations, and governances. It is due to the fact that both of these concepts may facilitate cost effective and wireless communication [4]. One of the most important and notice worthy benefits, which are expected to be provided by ad hoc networks and cloud based services, is cost efficiency and effective communication, which is one of the core factors in smart cities. It is assumed that, both ad hoc networks and cloud based services are expected to minimize or reduce the risk of network outages. In the current era of fast paced corporate environment, network outages are considered as one of the most devastating issues, as it impacts or influences the operations and functions of organizations. In this scenario, both approaches, i.e., ad hoc networks and cloud based services may commendably minimize or control network outages. In addition to this, both of these approaches may play an integral role in attaining the goal of smart governance and smart cities [2]. It is because; these 
concepts will provide highly integrated, effective, timely and cost effective wireless communication. This wireless communication will help the citizens as well as the government entities to immediately respond to the vulnerable and emergency situations.

It has been declared by S. Taneja [6] that ad hoc networks and cloud based services will also help in reducing the environmental susceptibilities, as both of these apaches eliminates the need of excessive energy, i.e., electricity. In this regard, G. Mcgilvary [5] has stated that cloud based services and ad hoc networks can be categorized as one of the greatest initiatives towards smart governance, smart cities, and sustainable and healthy environment. It is due to the fact that it reduces IT costs, slashing emissions of carbon, as well as use of electricity, while improving mobility and wireless communicatio [4]. More so, both of these networking techniques also opens new doors of telecommuting (i.e., through e-mails and internet), which brings added smart governance and smart cities benefits. In other words, it can be affirmed that ad hoc and cloud, jointly, holds a guarantee to reduce cost, improving continuous, uninterrupted, secure, and wireless communication, in order to develop and establish smart cities as well as governance.

\section{Conclusion}

From above paper, it has been concluded that mobile ad hoc networks has considerable importance in the growth and development of cloud based services. It is due to the fact that both of these networks comprise relatively same characteristics and features. Because of having the feature of excellent mobility, ad hoc network is capable to support and facilitate the establishment and growth of cloud based networks and services. It has been concluded from the foregoing paper that mobile ad hoc networks or MANET is the collection or set or group of various different independent users that coordinate and collaborate, by the help of wireless links. On the other hand, cloud computing also acquires similar properties, i.e. wireless communication within the predesigned network. It has been established from this research that ad hoc networks possess various issues and limitation, in terms of network privacy and performance. It has also been inspected that the challenges may considerably impact or influence the integrity and performance of the cloud based services. In order to overcome those challenges or performance limitation, different approaches and techniques have been discovered, in order to assure the reliable, uninterrupted, and flawless routing process; hence results in sustainable communication in cloud based services. This research study has discussed different issues as well as appropriate methods to overcome those issues.

\section{References}

[1] Kirby, G., Dearle, A., Macdonald, A., \& Fernandes, A. (Feb. 2010). An approach to Ad-hoc cloud computing. Eprint arXiv:1002.4738.

[2] Al-Omari, S. A. K., \& Sumari, P. (March 2010). An overview of mobile Ad-hoc networks for the existing protocols and applications. Int. J. Appl. Graph Theory Wirel. Ad-hoc Networks Sens. Networks, 2(1), 87-110.

[3] Hussain, R., Son, J., Eun, H., Kim, S., \& Oh, H. (2012). Rethinking vehicular communications: Merging VANET with cloud computing. Proceedings of 2012 4th IEEE International Conference on Cloud Computing Technology and Science (pp. 606-609).

[4] Mcgilvary, G. A. (2014). Ad-hoc cloud computing. School of Informatics, University of Edinburgh.

[5] Gupta, R. (2011). Mobile Ad-hoc network (MANETS) : Proposed solution to security related issues. Indian J. Comput. Sci. Eng., 2(5), 738-746.

[6] Taneja, S., \& Kush, A. (2010). A survey of routing protocols in mobile Ad-hoc networks. Int. J. Innov. Manag. Technol., 1(3), 279-285. 


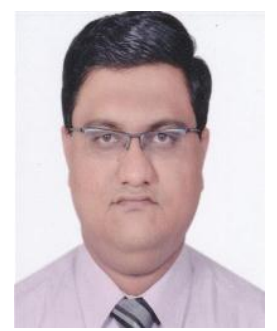

Aniket Deshpande is now pursuing his $\mathrm{PhD}$ degree in computer networking at Mewar University, Chittorgarh, Rajasthan, India under the guidance of Dr. Ashok Kaushal. He received his master of technology degree in computer science and engineering from Dr. MGR Educational and Research Institute, Chennai, Tamil Nadu, India. Prior to that he completed his bachelor of engineering degree in computer technology from Nagpur University. Network infrastructure optimization has been his key area of interest for last many years.

He is currently working with the Riverbed Technology as a system engineer. He has worked in the domain of networking strategy consulting as well as network infrastructure and application performance optimization during his nearly 7 years working experience with IBM in India. He also had significant exposure on VoIP technologies while working for Avaya. 